\title{
Survey on Cooperative Relay Selection Approaches
}

\author{
Nimmi Krishna M.R ${ }^{1}$, Shiras S. $\mathrm{N}^{2}$ \\ ${ }^{1,2}$ Department of Electronics and Communication, MBCET, Trivandrum, India
}

\begin{abstract}
Cognitive radio has been proposed in order to promote spectrum utilization by exploiting the spectrum holes. Observing the spectrum resources can be matched to traffic demand of secondary users with the help of relay node. In this article a new research direction for cognitive radio networks by utilizing cooperative relay to assist transmission and improve communication efficiency. Since wireless networks are characterized to have limited resources which are accessed by a large number of mobile towers with distinct capabilities. In such challenged environment the dynamic resource control is used to eliminate the limitations of wireless networks. Such augmented wireless resource usage can be incorporated based on cooperative relaying schemes, which have the capability to support the network lifetime and desired system performance. However, the introduction of cooperative relay raises several problems such as the relay selection issue and resource allocation. Due to the significant number of different cooperative relaying techniques, the aim of this survey is to provide a systematic and thorough analysis and classification of major relay selection procedures, and to identify research directions as well as the evaluation methods for an efficient analysis of different approaches.
\end{abstract}

Keywords: cooperative relaying; relay selection;spectrum utilisation ; network lifetime

\section{Introduction}

Cognitive radio is an emerging technology for future radio networks [1, 2], which enables an unlicensed cognitive user to recognize or to sense the spectrum and access a spectrum hole that is a radio frequency band licensed to a primary user but not used by that user. As the number of wireless terminals increases, higher system capacity is needed to provide the required data rate levels. Although wireless networks provide easy connectivity and fast deployment, they also present low performance levels. The limited resource devices, shared medium and unstable wireless channels are the major limitation of wireless networks. Channel conditions in wireless networks are subjected to fading variations, mainly the interference that can affect both throughput and reliability. As a result, receivers may get multiple copies of the transmitted signal, in which each of them are having travelled through a different path. Such multipath fading increases the number of errors in transmission, requiring additional re-transmissions that decrease the network throughput. The multipath fading effects can be eliminated by using cooperative communications, which aims to take advantage of wireless diversity to enhance efficiency levels.

The application of cooperative communications include ad-hoc self organizing networks and vehicular networks, sensor networks and dynamic spectrum management. When nodes have intermittent access to a network infrastructure, technological challenges increase due to the presence of low data rate stations and in mobile scenarios. In the former case, low-data rate devices may grab the radio spectrum for long periods of time which may decrease the network performance. In such situation high-data rate devices act as relays, which help the low-data rate devices to release the spectrum earlier, contributing to increase the overall system performance.

In this paper, the application of cooperative relaying, is focused to increase spectrum and power efficiency, network coverage and also to reduce outage probabilities. With the introduction of cooperative relaying, a special attention is required for relay selection since it has a strong impact on network and transmission performance.

This article provides an analysis of relay selection procedures proposed in recent years. The goal is to identify the best relay selection mechanism to support the design of cooperative MACs. This survey includes the creation of a taxonomy and analysis of open research issues.

\section{Cognitive Radio}

Cognitive radio networks are an innovative approach to wireless engineering were radios are designed with an unprecedented level of utility and intelligence. This advanced technology enables radio devices to use spectrum in entirely new sophisticated ways. The ability of cognitive radios is to monitor, sense, and detect the conditions of their operating environment, and dynamically reconfigure their own characteristics for best performance. 
Cognitive radios can identify potential impairments to communications for improving quality, like interference, path loss, shadowing and multipath fading. They ensure an optimized communications experience for users by adjusting their transmitting parameters, such as power output, frequency, and modulation.

Conventional radios have been designed with the assumption of operating in a spectrum band which is interference free. Thus there was no requirement to enable these radios with the ability to change parameters or spectrum bands in response to interference. Cognitive radios have been engineered from the basic level function to challenging conditions. In conventional radios, spectrum that is not being used is identified by viewing the environment in detail and quickly tune to that frequency to transmit and/or receive signals. They have the ability to instantly find other spectrum if interference is detected on used frequency.

Cognitive radio is a dynamically configurable device; it has the intelligence to respond to the surrounding radio frequency environment and is the key technology used to meet the requirements of the dynamic spectrum access. Cognitive radios are built on the software defined radio. These devices can achieve some requirements related to the spectrum sensing techniques through programming. It has the dynamic spectrum accessing capability (DSA).

Cognitive radios are different from other radios by its intelligence of functioning. The functioning of this device is categorized mainly in two phases 1) cognitive capability 2) Re-configurability by combining these two phases cognitive radio can work more efficiently and able to produce better performance.

\section{A. Some aspects of Cognitive Radio}

\section{A.1. Cognitive Capability}

Cognitive radio keeps a real-time interaction with its radio frequency environment. This adaptive feature of cognitive radio is taking some additional tasks. It has to take temporal and spatial measurement of RF environment [1] and also it should not make any interference free. By satisfying these requirements it has to select best spectrum.

\section{A.2. Re-configurability}

Re-configurability means the adaptability of cognitive radio according to the sudden change in the environment and ability to reconfigure its own parameters.

Operating frequency: It has the ability to change its own operating frequency according to the user requirement.

Modulation scheme: The modulation scheme must be changed by the cognitive radio depending upon the need of the user and the channel conditions.

Transmission power: Cognitive radio operates within a permissible power limit, so that this power can be changed according to the nature of application.

Thus Cognitive radio is an emerging technology to improve the wireless spectrum utilization. This allows a secondary user ( $\mathrm{SU}$ ) to access a licensed spectrum simultaneously with a primary user (PU)) as long as the interference from SU to PU is below a required threshold, i.e., the primary user's QoS is not affected. Hence, the transmit power of SU should be controlled to satisfy a given primary user QoS. However, when the primary user QoS is very strict, lower transmit power is allowed for secondary transmissions and the secondary throughput is limited. Cooperative diversity has been incorporated with cognitive radio to compact fading effect and enhance channel throughput.

\section{Cooperative Relaying}

Cooperative relaying is a advanced technique for wireless communications which promises gain in throughput and energy efficiency. The basic idea is that a device transmits a data signal to a destination and a third device overhears this signal and relays the signal to the destination. Finally, the destination combines the two received signals in order to improve decoding. This concept gives rise to pure wireless self-organizing networks. It can be employed in various applications of networked embedded systems, traffic systems. Cars use it to communicate directly with each other and can exchange reports on accidents, traffic jams, and about bad road conditions.

The devices cooperate to transmit signals over the environment and exploit a new, distributed form of spatial diversity that mitigates the negative effects of signal fading and interference. The task of relay selection is an important building block to realize cooperative relaying in practice. Devices located between sender and receiver must agree in a distributed manner so that it will act as relay and thus enhance wireless communication.

Most of the approach uses Markov chains to model and evaluate relaying protocols taking into account the achieved throughput, required energy and signaling traffic for communications. Cooperative relaying is important for industrial sensor networks. These networks require low-cost devices though highly reliable communication in heavy-cluttered environments. Cooperative relaying is a communication technique which 
improves link reliability between communicating entities. Cooperative relaying is used to improve reliability of wireless communications in fading environments [1]-[2]. It enables a relay node to support point-to-point transmissions between two source and destination nodes. The relay overhears source transmissions and forwards the data to the destination. Such a technique enhances space-time diversity. Many aspects of cooperative relaying have been investigated in the past years such as capacity limits, channel coding techniques, networking aspects and medium access protocols.

The concept of cooperative diversity is a means to eliminate the effects of small scale fading and exploits alternative communication paths by getting help from other nodes in the vicinity of sender and receiver of a currently affected communication link. These other nodes act as relays, i.e., a temporarily elected wireless node that assists in forwarding information from a source to destination nodes. The information relayed will flow establish a communication path concurrent to the direct communication path from source to destination or to communication via other relays. The alternative path is uncorrelated from the fading effects on the direct transmission path due to the spatial and time diversity.

\section{The key scientific goals of cooperative relaying include the following:}

- Relay selection: What are the decision criteria? When, and by whom and how is relay selection done?

- Cooperative MAC protocols: How do cooperative relaying can be integrated into a protocol stack?

Thus cooperative relaying has great potential to assist communication pairs in wireless networks by eliminating the effects of multipath fading. The benefit of cooperative relaying rely on broadcast nature of wireless networks where several nodes overhear ongoing communication between source-destination pair. Therefore, a copy of packet can be retransmitted by neighbouring node that is overheard the direct transmission, even if a packet cannot be delivered to a destination due to impaired channel conditions.

Relay selection is one of the main buildings blocks of cooperative relaying and commonly channel conditions of relay links are main selection criteria. The impact of choosing a given relay node on communication of surrounding nodes and overall network has to be taken into account.

\section{Relay Selection Approaches}

Relay selection is one of the major building blocks of cooperative relaying and it is the main criteria are the channel condition of relay links. The impact of choosing a given relay node on communication of surrounding nodes as well as the overall network also needs to be taken into account is also considered necessary.

The performance of cooperative relaying mainly depends on relay selection. The relay selection is mainly done once at network startup periodically at transmitting section. Many papers have been published based on different relay selection approaches.this paper gives a survey on relay selection approaches in cognitive radio networks.

In [7], Shan et. al. proposed to group neighbouring nodes according to their helping ability which is expressed by resulting cooperative rate. Here a group of node is used for cooperation is determined by performing a series of contentions. Thus obtain optimal nodes in the group and from this the best relay is selected. The selection mechanism gets terminated when the required optimal relay is obtained. H. Shan et. al. proposed in [4] a medium access control(MAC) protocol based on Carrier Sense Multiple Access with Collision Avoidance(CSMA/CA) that is included in cooperative relay selection.

All previous relay selection approaches assume that relays are always needed. K.-S. Hwang et al. [6] proposes an opportunistic approach in which relay selection is only triggered by the destination when the estimated (by the destination) CSI is lower than a pre-defined threshold. This reduces energy consumption. However relay selection still relies on overhearing RTS and Clear-to-Send (CTS) frames, leading to an increase in the communication overhead, especially in multi-hop scenarios.

In all previous approaches relays are selected in such a way that the relays do not know the channel information of other potential relays. Sources select either a direct transmission or the transmission through a relay by look-up into the local table. However, the relay selection is based on historical information and may not work in mobile environments.

N. Marchenko et al. [12] proposed a method to select a relay selection based on spatial efficiency and channel conditions, which is achieved when the relay selection results in few additional transmissions being blocked. Although selecting relays closer to source reduces the probability of blocking other transmissions in terms of spatial efficiency, it decreases the benefits brought by spatial diversity.

The use of nodal location information is beneficial in communication network protocols. Zorsi and Rao in [9] use location of nodes for forwarding data from source to destination in multihop networks. The forwarding node closer to destination is selected at each hop after transmission. Groups are built by nodes in the network according tto distance in destination. The group members participate in contention by receiving the message. 
An analysis of relay selection procedures proposed in recent years in this article. The aim is to identify the most suitable relay selection mechanism to support the design of cooperative MACs and cooperative routing mechanisms. This study involves the performance analysis of the most prominent proposals and the creation of taxonomy. This article ends with an analysis of open research issues.

In what concerns relay selection mechanisms, the basic mechanism proposed in [10] defines an opportunistic behavior in which overhearing nodes estimate their Channel State Information based on which they set a timer such that nodes with better channel conditions broadcast first their qualification as relays, or even data to be relayed. Another simple proposal [11] uses the measured Signal-to-Noise ratio (SNR), the overhearing nodes send out a busy-tone (the relay with best channel conditions sends longer busy tone). Such mechanisms present a high probability of collisions. The opportunistic relaying has been modified aiming to increase its efficiency [4], [5], [6].

Tao Jing et. al. [1] proposed an optimal stopping theory for cooperative relay selection. As the number of secondary users may be large it is infeasible to scan all candidate relays, thus optimal stopping rule is applied early enough to avoid scanning all the relays. Here an optimal observation sequence is also considered based on decreasing order of data rate. Thus the problem of relay selection is carried out i.e., how to efficiently find an appropriate relay that can satisfy primary transmitter's quality of service.

\section{Taxonomy of Relay Selection}

The major challenge in cooperative relaying is to select a node, or set of nodes, which can effectively enhance data transmission. Since most of the current schemes envision operation under a single AP, there should be careful relay selection mechanisms definition which think about large networks. One of the reason is unsuitable performance of chain relaying processes along multiple hops. Other reason in the impact that one relay may have on concurrent transmissions.

Selection criteria are the first aspect that needs to be considered when analyzing relay selection mechanisms. As seen in section IV, the most common in the literature are parameters like CSI, SNR and PER. Since such parameters need to be measured in sender-relay and relay-receiver links, relay selection may require the exchange of meta-data, usually transported using RTS and CTS frames.

The overall network is the second aspect. Normally relays are selected to improve the performance of a source-destination communication (e.g. [2], [9]), but no consideration is taken about the impact over the overall network capacity. Such behavior leads to higher probability of transmission blocking and interference.

For a better understanding of the utility of current relay selection approaches, they are classified based on the time of selection (three classes) and level of interaction (two levels), as shown in Figure 1.

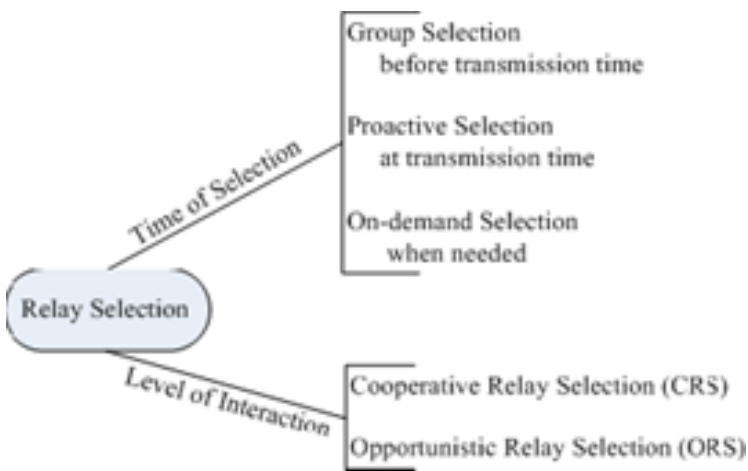

Figure 1. Relay selection taxonomy

Based on the time of selection, the following are the three categories:

- Group selection, in which relay selection occurs before transmission which aims to achieve certain predefined performance level.

- Proactive selection, in which relay selection is carried out by the source, the destination, or the relay itself at transmission time.

- On-demand selection, in which relay selection is carried out when needed, namely when direct channel conditions decrease below a pre-defined threshold.

Based on the level of interaction, relay selection mechanisms are grouped into two categories:

- Opportunistic Relay Selection, here based on the information that it has about the network each potential relay decides about forwarding packets. This may lead to a high probability of selecting more than one relay whose transmissions will compete for the wireless medium. 
- Cooperative Relay Selection, process encompasses two phases: In the first phase relays broadcast acceptance to relay and local information that will be useful for relay selection. Such information is overheard by other nodes, which participate in the selection of one or more relays in a second phase. One of the drawbacks of cooperative relay selection is the lack of synchronization between the two operational phases. As a consequence, the packet relaying will not occur if a node that was selected as relay is not available when transmission is required, due to mobility or lack of energy.

For a better understanding of the existing relay selection proposals, seven types of relay selection approaches are analyzed, being the classification based on the taxonomy presented in section III (Figure 2). This survey is helpful to identify similarities among existing proposals, supporting the decision about the relay selection methods that better suits novel cooperative relaying proposals.

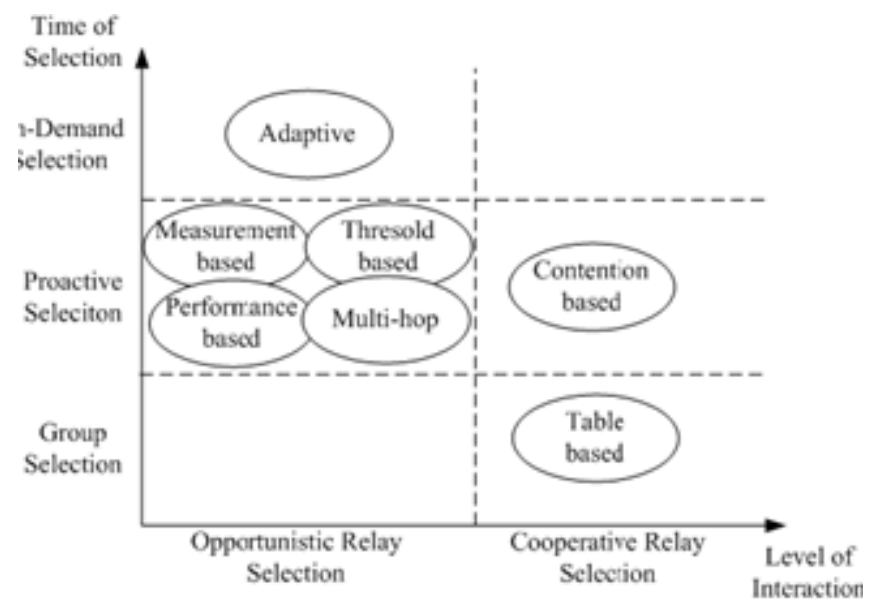

Figure 2. Classification of Relay Selection Approaches

Based on the figure 2, the basic opportunistic relay selection scheme is based on local measurements. Many other approaches aim to eliminate the limitations of measurement-based relay selection, by minimizing the overall transmission power which is by, performance-based relay selection and the channel estimation overhead which is done through threshold-based relay selection. The three approaches mentioned here are opportunistic and follow a proactive selection approach, which means that a relay is always selected. The adaptive relay selection which is also an on-demand selection category follows a different technique, in which only if needed the relay selection procedure is triggered.

The exchange of information is required for the cooperative relay selection procedures among the involved communication nodes. In this case two categories are identified. One that leads to the selection of a controlled number of relays based on information kept by the source, called table-based relay selection and a second category that leads to the selection of a set of a variable number of relays called contention-based relay selection. In this case by making use of the contention windows competition among relays may be reduced.

All mentioned relay selection approaches consider transmissions over a direct source destination link only. This assumption will restrict the scenarios over which cooperative relaying may be used. To expand the utility of cooperative relaying to multi-hop networks an extra category called multi-hop relay selection is considered in which relays may also help source-destination communications even when such communications have to go other an intermediary nodes.

\section{A. Measurement-based Relay Selection}

Measurement-based relay selection approaches are characterized with no topology information, and is based only on local measurements of instantaneous channel conditions. The opportunistic relaying approach proposed by A. Bletsas et al. [2]. Shan et al. [3] is another example.

In general the operation of measurement-based selection approaches is as follows: each potential relay estimates channel conditions (CSI in case of [2]) of source-relay and relay destination channels by using Ready To Send and Clear To Send signaling. CSI estimation is based on fading amplitudes between source-relay and relay-destination and on the required performance of the source-relay-destination channel. Each relay sets a transmission timer to an inverse value to the estimated CSI value after CSI estimation. The timer with the best suitable CSI ceases earlier, qualifying that device as relay. When the devices in listening mode overhear a short duration packet sent by the qualified relay, it will back off as soon as possible. The qualified relay may request the destination to notify all the other potential relays about its transmission to avoid the case of "hidden" relays.

Measurement-based approaches select the best relay among $\mathrm{N}$ devices, and for this they may require $2 \mathrm{~N}$ channel state estimations, which is in the same complexity order as conventional Distributed Space-Time 
Coding (DSTC) algorithms [15]. DSTC algorithms in order to support complex signal processing at receiver require significant modifications of hardware.

\section{B. Performance-based Relay Selection}

The performance-based selection approaches rely on performance criteria mainly on delay and energy efficiency. It is done to select the most suitable relay, aiming to optimize measurement-based approaches. The examples of performance-based relay selection are the approaches proposed in [5] and [7].

In general the operation of performance-based selection approaches is as follows: The sources transmit their required performance level in first phase, and all potential relays estimates their channel conditions as well as performance level in second phase. However, estimation overhead may bring some limitations to performance-based approaches, and the transmission may still occur over the direct link if the performance conditions are not met.

\section{Threshold-based Relay Selection}

The threshold-based approaches rely on a certain threshold to reduce the number of competing relays, and thus reduce the overhead of channel estimations. This category of relay selection can follow any category of interaction (c.f. Figure 1). The relay selection involves mainly two phases. In general the operation of thresholdbased selection approaches is as follows: each neighbor compares the quality of signal it received from the source with a threshold such as SNR (in case of [4]) or BER (in case of [8]) in first phase. Only relays which satisfies the threshold requirements will enter into relay selection according to the algorithm in second phase. For instance, the node with the maximum lower value of the SNR in the source relay and relay-destination links is selected as a relay in [4].

\section{Adaptive Relay Selection}

The PER of the link from source to destination may decrease due to variations on channel conditions in a way that relaying over a helping node is not needed. Adaptive relay selection approaches perform relay selection only if relaying is needed with high probability. An example of adaptive relay selection is Adam et al. [24]. In general the operation of adaptive relay selection approaches is as follows: in a first phase the destination compares the quality of received signal with a pre-defined threshold. If the quality of received signal is below the specified threshold, then the relay selection process is triggered. In case of the Adam et al. [24] relay is chosen similar to basic opportunistic approach proposed in [2]. The adaptive schemes should address the transmission collision problem and more advantage of spatial diversity should be obtained. Moreover, thresholds at destination should be optimal to guarantee fast reaction to channel variations.

\section{E. Table-based Relay Selection}

The table-based approaches follow a cooperative relay selection process aiming to decrease relay selection impact on transmission time. In general the operation of table-based approaches such as CODE and CoopMAC is as follows: sources keep CSI information of the links between themselves and potential relays as well as of the links from potential relays and each potential destination. Using RTS/CTS frames as well as information collected from overheard transmissions the CSI information is gathered. Relays are selected by looking up in a table by the source. A node is selected as relay if the transmission time over the direct link to a destination is higher than the sum of the transmission time over the source relay and relay-destination links. The major difference between CoopMAC and CODE is that with the latter, a source selects two relays with latest feedback time, forming a cooperative diamond. Here the usage of RTS-CTS frames is also different. Code proposes the cooperative-RTS signal which is sent by the source to the relays and by the latter to the destination to show their acceptance to cooperate. If the destination finds that such cooperation is beneficial via both relays, it sends a cooperative- CTS signal to the source. Table-based approaches present degradation problems in the presence of moving nodes. Another problem with this category is the periodic broadcast and extra handshaking signals which can limit the communication efficiency.

\section{F. Multi-Hop Relay Selection}

The most common relaying approach in the literature is to select a relay to help a transmission from a sender to a destination over a poor direct wireless link. In case of multi-hop networks, this method requires the repetition of the relay selection procedure for each hop from sender to destination. However, the network capacity is reduced by such hop-wise cooperation. One solution is to select relays that can help more than one link simultaneously Lichte et al. [7], Adam et al. [24]. Such higher diversity is not possible to obtain with 802.11 MAC protocols as they are not aware of following hops. In general the multi-hop relay selection approaches operation is as follows: routing information is accessed by potential relays creating a limited image of the network beyond the adjacent wireless links. By overhearing transmissions over the identified network, 
potential relays decide to relay overheard information to potential destinations, even in the absence of a direct link between the source and destination. This means that relays may have received the information to be relayed directly from the source or from other relays or intermediary nodes. In multi-hop relay selection, the destination node will receive more than two independent signals of the same packet. This extra spatial diversity increases performance and robustness. However, the cost to pay is the extra network overhead to transmit redundant information will not be updated with the frequency required to react in environments with mobile devices.

\section{Observations and Open Research Issues}

From the realized study given above, two strong observations can be made: i) all approaches assume small networks, static devices with high probability or a direct source-destination link usage, and the need to use always one relay; ii) there is no single approach and both transmission and network performance presents the good behavior. Here approaches try to increase network lifetime or transmission throughput. Multi-hop relay selection approaches are one exception. However, performance of multi-hop approaches depends upon the existence of link-state routing. The mentioned observations lead to the identification of two important research issues: i) achieve a good balance between transmission throughput and network lifetime; ii) improve the capacity of large mobile networks. Before investigating suitable solutions to achieve a good balance between network lifetime and transmission throughput, following question has to be answered: when do there is really a need to use cooperative relaying? To answer this question the network lifetime and transmission throughput achieved that take advantage of cooperative relaying have to be compared and by techniques that take advantage of time diversity over the direct link. The CSI accuracy levels can be ensured in relation to different levels of channel variation in order to devise a cooperative relay solution which is able to obtain a good balance between network lifetime and transmission throughput. Moreover, although all analyzed approaches make use of RTS/CTS mechanism, it would be important to investigate approaches related to RTS/CTS, aiming to reduce overhead.

In order to investigate the most suitable solutions to improve the capacity of large mobile networks, two basic assumptions are taken: i) mostly devices are mobile; ii) connectivity may be intermittent. The majority of the analyzed approaches assume situations where nodes are static and so that it is not difficult to select a direct link between source and destination. In the case of scenarios with multiple hops between source and destination, it is better to exploit the multi-hop relay selection, namely trying to identify the most suitable relay/hops ratio. The current multi-hop relay selection approaches rely on link-state routing information, which means that they are not suitable for scenarios with intermittent connectivity. Hence the investigation of the usage of multi-hop relay selection in the presence of opportunistic routing is an important research topic.

\section{Future Direction On Relay Selection}

The observations obtained from the previous section indicate that cooperation provide benefits to the operation of wireless networks but its usage over large networks causes undesirable levels of overhead and complexity. The undesirability is mainly due to the number of channel estimations, while the overhead is mainly due to the multiple copies of informations and feedback signals.

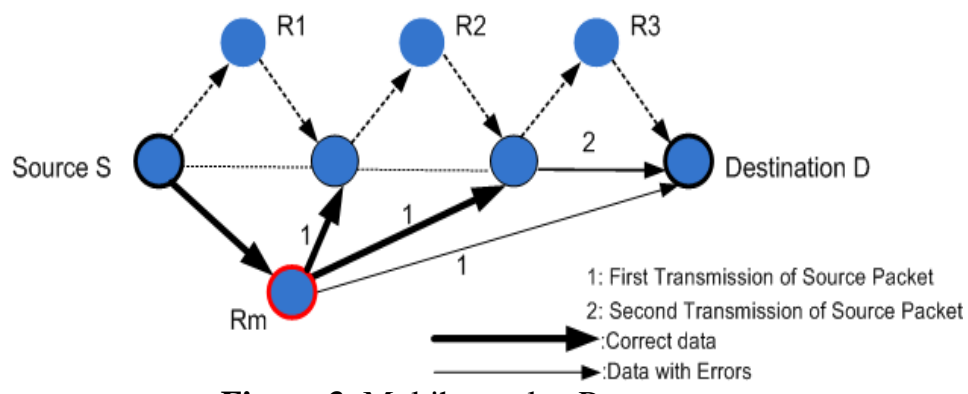

Figure 3. Multihop relay $\mathrm{Rm}$

The complexity may increase in mobile scenarios due to the number of times relay selection must be performed due to frequent link degradation. The waiting for optimal relay to assist one transmission degrades the overall performance of the network and decreases its capacity. The presence of dense networks will also bring performance degradation, due to the high interference levels. Here the interference caused by relay transmissions will be, in the best case is directly proportional to the relay degree. The situation will get worse in the presence of multi-hop networks, where the usage of hop by-hop cooperation will increase the network cost. Figure 3 shows that by wisely selecting a relay to assist several transmissions in a multi-hop scenario (Rm) and can reduce the number of relays $(\mathrm{Rm}, \mathrm{m}=1,2,3)$ and consequently the number of transmissions. 


\section{A. Opportunistic-Cooperative Relay Selection}

A major conclusion of investigation is that to avoid extra overhead and complexity, relays should be selected with limited feedback in a distributed manner and by computing just local parameters. In addition interrelay cooperation should be taken into account in order to improve spatial reuse, but cooperation and exchange of information produces overhead and energy consumption. So that the usages of opportunistic relay selection schemes is a good choice in a collision free environment. But in a realistic scenario, scheduling and priorities are important to consider, which provide some cooperation level that needs to be taken into account.

Once a node or set of nodes are selected as a relay, they should cooperate with the source, destination or even with other selected relays dynamically, in order to achieve better results with varying network conditions. This will give rise to a new level of interaction called "Opportunistic-Cooperative Relay Selection", where multiple relays may have different priorities.

\section{B. Evaluation Parameters}

To limit communication overhead, mainly in large networks, it is important to investigate the intelligent usage of threshold over local variables, as they can filter out poor relays as well as unwanted transmissions. Based on the type of thresholds, they should be adaptive to cope with the varying nature of networks.

The previous work uses parameters such as local variables such as SNR, BER, CSI [21]. Since these are very unstable parameters, the usage of energy levels, interference level and stability is considered. Node degree and queuing delay are examples of parameters that can be used to estimate the interference level, without using physical layer measurements. Energy is an important parameter, mainly in battery constraint networks. The last parameter, stability, is very most important and has not been considered by any prior work.

Stability is the measure of mobility, and can be obtained by estimating pause time or link duration. The more stable nodes are, the more suitable are they to operate and relays. So, investigation lead to the conclusion that the most suitable relay selection scheme for large scale networks is devised by using local parameters such as SNR, BER and CSI parameters.

\section{Summary and Conclusion}

This article provides an analysis of relay selection approaches for wireless cooperative networks, since a poor selection can degrade the performance of the overall network. A taxonomy for the evaluation of relay selection approaches, and an analysis of their performance. Some observations which are provided about topics that need to be further analyzed to devise cooperative relaying systems able to optimize concurrent communications in huge networks composed of mobile stations. Based on a systematic and thorough analysis of relay selection approaches the initial conclusions are of three-fold: i) to achieve a good balance between the performance of individual transmissions and performance of the overall network relay selection should support the systems; ii) relays should be selected based on stable parameters like signal to-noise ratio or packet error rate, the usual channel state should be avoided; iii) multi-hop scenarios as well as scenarios with mobile nodes should be supported with good relay selection schemes. The usage of such cooperative relay selection scheme will provide the needed distributed intelligent to support relaying over large networks.

\section{References}

[1]. Tao Jing,Shixiang Zhu,Hongjuan Li,Xiaoshuang Xing,Xiuzhen Cheng,Yan Huo,Rongfang Bie,and Taieb Znati, “Cooperative relay selection in cognitive radio networks," IEEE Trans. On Vehicular Technology,vol.64,NO.5,May 2015.

[2]. H. Shan, W. Z. P. Wang, and Z. Wang, "Cross-layer Cooperative Triple Busy Tone Multiple Access for Wireless Networks," in Proc. of IEEE Globecom, New Orleans, USA, Dec. 2008.

[3]. A. Bletsas, A. Khisti, D. Reed, and A. Lippman, “A simple Cooperative Diversity Method Based on Network Path Selection,” IEEE Journal on Selected Areas in Communications, vol. 24, no. 3, pp. 659-672, Mar. 2006

[4]. J. Morillo-Pozo, O. Trullols, J. M. Barceló, and J. García-Vidal, "A Cooperative ARQ for Delay-Tolerant Vehicular Networks," in Proc. Of IEEE ICDCS, Beijing, China, Jun. 2008

[5]. K.-S. Hwang and Y.-C. Ko, “An Efficient Relay Selection Algorithm for Cooperative Networks,” in Proc. of IEEE VTC.

[6]. Y. Chen, G. Yu, P. Qiu, and Z. Zhang, "Power-Aware Cooperative Relay Selection Strategies in Wireless Ad Hoc Networks," in Proc. of IEEE PIMRC, Helsinki, Finland, Sep. 2006.

[7]. H. S. Lichte, S. Valentin, H. Karl, I. Aad, L. Loyola, and J. Widmer, "Design and Evaluation of a Routing-Informed Cooperative MAC Protocol for Ad Hoc Networks," in Proc. of IEEE INFOCOM, Phoenix, USA, Apr. 2008.

[8]. Z. Zhou, S. Zhou, J. Cui, and S. Cui, "Energy-Efficient Cooperative Communications based on Power Control and Selective Relay in Wireless Sensor Networks," IEEE Journal on Wireless Communications, vol. 7, no. 8, pp. 3066-3078, Aug. 2008.

[9]. J. Mitola and G. Q. Maguire, “Cognitive Radio: Making Software Radios More Personal,” IEEE Pers. Commun.,vol. 6, 1999, pp. $13-18$

[10]. M. Song, C. Xin, Y. Zhao, and X. Cheng, "Dynamic spectrum access: From cognitive radio to network radio," IEEE Wireless Commun., vol. 19,no. 1, pp. 23-29, Feb. 2012

[11]. W. Elmenreich et al., "Building blocks of cooperative relaying in wirelesssystems," Elektrotechnik und Informationstechnik, vol. 125, no. 10,pp. 353-359, Oct. 2008.

[12]. Y.Zou, B. Zheng, W.-.P. Zhu, and J.Cui, "An optimal relay selection scheme for cooperative diversity", in proc. IEEE ICSP 2008, Beijing China, Dec. 2009. 
[13]. N. Marchenko, E. Yanmaz, H. Adam, and C. Bettstetter, "Selecting a Spatially Efficient Cooperative Relay," in Proc. of IEEE GLOBECOM, Honolulu, Hawaii, Nov. 2009.

[14]. Y. Li, P. Wang, D. Niyato, and W. Zhuang, "A dynamic relay selection scheme for mobile users in wireless relay networks," in Proc. IEEE INFOCOM, Apr. 2011, pp. 256-260

[15]. J. Lai, E. Dutkiewicz, R. P. Liu, and R. Vesilo, "Comparison of cooperative spectrum sensing strategies in distributed cognitive radio networks," in Global Communications Conference (GLOBECOM), 2012 IEEE, 2012, pp. 1513-1518.

[16]. J. N. Laneman and G. W. Wornell, "Distributed SpaceU" Time-Coded Protocols for Exploiting Cooperative Diversity in Wireless Networks,'IEEE Transactions on Information Theory, vol. 49, no. 10, pp. 2415-2425, Oct. 2003.

[17]. T. Jing, X. Chen, Y. Huo, and X. Cheng, "Achievable transmission capacityof cognitive mesh networks with different media access control," in Proc. IEEE INFOCOM, Mar. 25-30, 2012, pp. 1764-1772

[18]. A. S. Ibrahim, A. K. Sadek, W. Su and K. J. R. Liu, "Cooperative communications with relay selection: When to cooperate and whom to cooperate with ?", IEEE Trans. Wireless. Comm., Vol. 7, No. 7, pp. 2814-2827, Jul. 2008.

[19]. J. Jia, J. Zhang and Q. Zhang, "Cooperative relay for cognitive radio networks", in proc. IEEE International Conference on Computer Communications (INFOCOM), pp. 2304-2312, Rio de Janeiro, Brazil, Apr.2009.

[20]. D. B. da Costa and Sonia A"1ssa, "Performance analysis of relay selectiontechniques with clustered fixed-gain relays", IEEE Sig. Proc. Lett., Vol.17, No. 2, pp. 201-204, Feb. 2010.

[21]. Q. Zhao and B. Sadler, "A Survey of Dynamic Spectrum Access,” Signal Processing Magazine, IEEE, vol. 24, no. 3, pp. 79-89, 2007.

[22]. S. S. Ikki and M. H. Ahmed, "Performance of multiple-relay cooperative diversity systems with best relay selection over Rayleigh fading channels", EURASIP J. on Adv. in Sig. Proc., Vol. 2008, Article ID 580368.

[23]. H. Sun, A. Nallanathan, C.-X. Wang, and Y. Chen, "Wideband spectrum sensing for cognitive radio networks: a survey," Wireless Communications, IEEE, vol. 20, no. 2, pp. 74-81, 2013.

[24]. H. Adam, C. Bettstetter, and S. M. Senouci, “Adaptive Relay Selection in Cooperative Wireless Networks," in Proc. of IEEE PIMRC, Cannes, France, Sep. 2008. 